\title{
Testing the Phylogeny of Swordtail Fishes Using Split Decomposition and Spectral Analysis
}

\author{
Peter J. Lockhart, ${ }^{1}$ David Penny, ${ }^{1}$ Axel Meyer ${ }^{2}$ \\ ${ }^{1}$ School of Biological Sciences, Private Bag 11222, Massey University, Palmerston North, New Zealand \\ ${ }^{2}$ Department of Ecology and Evolution, and Program in Genetics, State University, New York, StonyBrook, NY 11794-5245, USA \\ Received: 11 November 1994 / Accepted: 12 April 1995
}

\begin{abstract}
We examine ways of testing for the reliability of inference from biological sequence data using sequences from Xiphophorus fishes and newly implemented methodology for sequence analysis. The approach we take provides one means to examine the fit between model and data for different sequences and hence to evaluate heterogeneity between data sets. In the case of the present study we show D-loop sequences to be a better molecule for studying the phylogeny of $X i$ phophorus fishes than cytochrome $b$ sequences. The results of the split decomposition and spectral analysis confirm an earlier phylogenetic hypothesis which had been based on maximum parsimony, neighbor-joining, maximum likelihood analyses.
\end{abstract}

Key words: Spectral analysis - Split decomposition - Hadamard - Cytochrome $b-\mathrm{D}$-loop $-X i$ phophorus

\section{Introduction}

Reliable phylogenetic inference from biological sequence data is crucial for studies in molecular systematics. The desire to understand and improve the performance and test the limitations of evolutionary reconstruction methods with real biological data is a common theme in many recent studies (e.g., Golding 1993;

Correspondence to: A. Meyer
Olsen and Woese 1993; Steel et al. 1993a; Hillis et al. 1994; Lake 1994; Lockhart et al. 1994; Simon et al. 1994; Yang et al. 1994).

One new approach to the analysis of biological sequences termed "spectral analysis" (Hendy and Penny 1993; Hendy et al. 1994) emphasizes the identification of signals (patterns) in the data that may arise from different causes (e.g., Penny et al. 1993; Lento et al. 1995). This approach provides the means to examine such patterns when different corrections for unobserved multiple substitutions are made and hence to determine the usefulness of a given transformation to correct for these. The success of such transformations is measured in terms of their ability to resolve conflicting signals which would support competing internal edges in reconstructed evolutionary trees. With real biological data and as illustrated in the simple case of four taxa there is often some support in the spectra for all three possible binary, unrooted trees (Fig. 1). However, after an appropriate transformation to correct for multiple substitutions has been made the expectation is that the correct net phylogenetic signal (support-contradictions) will increase (Fig. 1).

In this paper we use spectral analysis to reexamine a recent controversy (Meyer et al. 1994; for reviews see Pomiankowski 1994; Shaw 1995) on the role of sexual selection in the evolution of sword-like caudal-fin extensions in males of some species of swordtail (in the genus Xiphophorus) fishes. In doing this we demonstrate how this approach can be used to (1) examine possible heterogeneity in phylogenetic signal between different genes (Huelsenbeck et al. 1994) and (2) address the issue of how to objectively determine which sequence data 

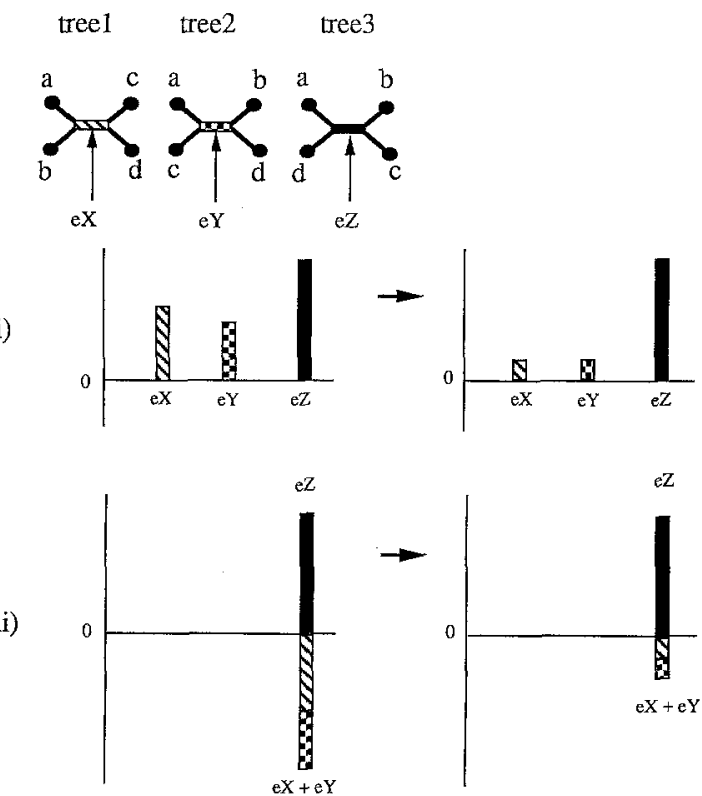

before correction

after correction

Fig. 1. With four taxa the three possible binary unrooted trees are each identified by a unique central edge (i.e., the branch or internode segment between two nodes), these have been labeled $e X, e Y$, and $e Z$. With spectral analysis two possible ways of representing the support in the data have been shown. In (i) the amount of support for each of these is represented as a histogram whose relative heights indicate their relative weights. In (ii) the support for each particular hypothesis is plotted above the zero point on the $\mathrm{X}$-axis. Below the line is plotted the sum of the contradictory support (i.e., sum of the weights for $e X$ and $e Y$ ). When corrected appropriately for multiple substitutions the expectation is that false support in the data for the incorrect tree(s) will be removed. Such corrections for multiple hits are done on the observed "paths" (observed differences) between taxa. A Hadamard transformation (discrete Fourier transformation; Hendy et al. 1994) is then used to add and subtract these paths to get values for the individual edges which make up the paths.

sets will provide the most reliable information for testing specific and different biological hypotheses (Meyer 1994a; Simon et al. 1994).

Morphological characteristics divide the genus Xiphophorus into swordtails and platyfishes. Each of these groups is also further divided into a southern and a northern group. However, these common names are somewhat misleading because although most platyfishes do not possess swords, some do have small swords similar in length to those of some of the swordtail species. The northern platies group is composed of three species ( $X$. couchianus, $X$. gordoni, $X$. meyeri), the southern platies group of six species ( $X$. andersi, $X$. variatus, $X$. milleri, $X$. maculatus, $X$. evelynae, $X$. xiphidium), the northern swordtail group of nine species $(X$. continens, $X$. multilineatus, $X$. nigrensis, $X$. pygmaeus, $X$. birchmanni, $X$. malinche, $X$. cortezi, $X$. montezumae, $X$. nezahuacoyotl), and the southern swordtail group of four ( $X$. clemenciae, $X$. alvarezi, $X$. signum, $X$. helleri). Based on a phylogeny largely reconstructed from morphological characters and
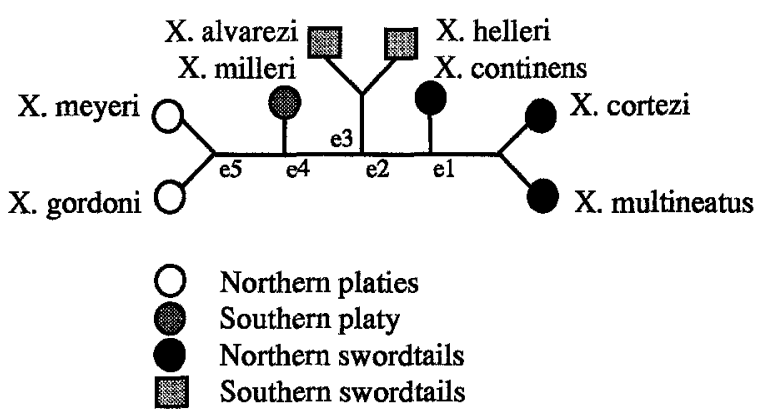

Fig. 2. Unweighted unrooted tree for a phylogeny between eight Xiphophorus species which are used in the present study and for which phylogenetic relationships are undisputed. Internal edges in the tree are labeled $e 1$. . e5. Support for these edges will arise as a result of patterns in the data which partition the taxa into different groups. For example, if at a given site in the sequence, $X$. meyeri and $X$. gordoni shared an " $A$ " residue and the other taxa all shared a " $G$," then under simple or no transformation such a pattern is likely to support the existence of edge $e 5$. If at a different site, the observed pattern partitioned the taxa X. meyeri, X. gordoni, and X. milleri from the other taxa, then this is likely to support the existence of edge $e 4$. The greater the number of such patterns in the data, the more support there will be for respective internal edges.

behavioral experiments with a southern platy and a southern swordtail species it has been suggested that the sworded condition in males evolved late in the evolutionary history of species of the genus Xiphophorus in response to preexisting female preferences to mate with males with swords (Basolo 1990).

To test the specific role of the sensory exploitation model in sexual selection for the evolution of the exaggerated secondary male characteristics, Meyer et al. (1994) recently presented the first molecular phylogeny for the Xiphophorus genus. This was based on 1,284 base pairs (bp) for 52 specimens of all 22 Xiphophorus species and six outgroups (from the genera Priapella, Poecilia, and Alfaro; of these Priapella was identified to be the sister to Xiphophorus). In their study, the correct placement of the outgroups was very important since the placement would identify the direction of evolution between the sworded and swordless conditions. Their data consisted of partial mitochondrial D-loop (402 bp) and cytochrome $b$ sequences (360 bp) and nuclear DNA sequences ( $522 \mathrm{bp}$ of the tyrosine kinase gene $X$-src). Each data set was analyzed separately and combined using the maximum parsimony (Swofford 1993) and neighborjoining methods (Saitou and Nei 1987). On the joint data set both methods yielded almost identical phylogenetic estimates. These placed the outgroups on an edge (internode) which separated the northern and the southern swordtails (e.g., edge $e 2$ in Fig. 2). On the assumption that the root of the tree should join the outgroup edge, this placement of the outgroups suggested that the sworded condition was in fact ancestral for the genus. This reconstruction contradicted the phylogeny used by Basolo (1990), which was based largely on morphological characters, and which suggested that the root of the 
tree was between the southern and northern platyfishes (Rosen 1979; Rauchenberger et al. 1990; e.g., the placement would be on edge $e 5$ in Fig. 2). This earlier phylogeny had suggested (1) that the platyfish were paraphyletic with the northern platyfish being most ancestral and (2) that the southern platies were more closely related to the swordtails than to the northern platies. In contrast, the molecular phylogeny of Meyer et al. (1994) placed the root between the northern and the southern swordtails. This suggested swordtails were ancestral and suggested that the platyfish are more derived than the swordtails. Since the correct evolutionary tree is essential for evaluating the applicability of the sensory exploitation hypotheses of swords in male swordtail fishes, here we examine the reliability of the molecular phylogeny reported by Meyer et al. (1994).

\section{Materials and Methods}

In the first aspect of this study we characterize the nucleotide substitution properties in the two most variable data sets which comprised the joint data set studied by Meyer et al. (1994). These were the cytochrome $b$ and D-loop sequences. The nuclear $X$-src data set they used did not have sufficient variability to study the phylogeny separately since these data contributed a relatively small number of patterns to the overall joint data set.

In order to allow a more detailed study of the sequences used for reconstructing the Xiphophorus phylogeny we evaluated the nucleotide substitution patterns for a subset of taxa from the different clades for which their phylogeny is undisputed-that is, those taxa whose relationships are identically described from both the morphological and molecular study. Figure 2 shows the relationship between eight such taxa. For this tree, we first investigate how well patterns in the cytochrome $b$ and D-loop sequences suggest the underlying phylogeny. We use only third codon position data in the case of cytochrome $b$ since most of the patterns in the Xiphophorus cytochrome $b$ sequences which indicate support for internal edges in reconstructed trees are in the third codon position. There are 120 sites in the third codon position. Of these sites, 35 vary with 19 of those exhibiting parsimony patterns (i.e., phylogenetically informative sites). No sites in the second codon position vary and only two sites in the first codon position show changes. In the eight taxon D-loop data set there are 402 sites. Of these, 43 positions vary and 34 of these are parsimony patterns.

Split decomposition analysis (Bandelt and Dress 1992) for these eight taxa was carried out using SPLITSTREE V 1.0 (Huson and Wetzel 1994) for both data sets. Hamming distances (observed number of differences/total sequence length) were used for these calculations (Fig. 3).

The "support" (relative partition size as measured by spectral values) which corresponds to each individual internal edge (e1-e5; Fig. 2) has also been calculated for Hamming distances and three transformations which may describe sequence evolution: Jukes-Cantor distances, Kimura 2ST distances, and LogDet values (Steel 1994; Lockhart et al. 1994). The corrected partitions, calculated from these path measures using a Hadamard method (Hendy et al. 1994), are shown as a spectrum in Figs. 4 and 5. The Jukes-Cantor and Kimura 2ST objective distances were calculated using DNADIST3.5 (Felsenstein 1993) while PREPARE (Penny et al. 1993) was used to calculate the Hamming distances and LogDet values. Spectral values were calculated using the program HADTREE (Penny et al. 1993). Variances on the spectral values have not been calculated. However, support for the partitions (which correspond to internal edges e1-e5 within both the eight taxon cytochrome $b$ and Dloop data sets) has been evaluated by bootstrapping
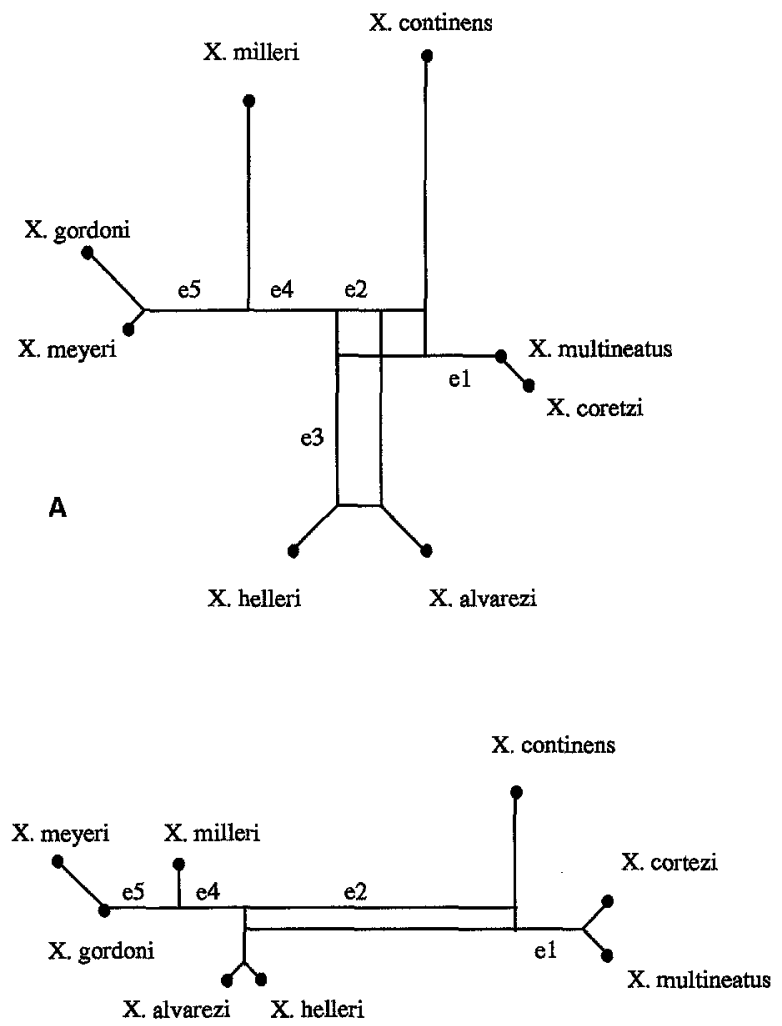

B

Fig. 3. SPLITSTREE graphs for cytochrome $b$ (A) and D-loop (B) sequences for the eight taxa also shown in Fig. 2. The graphs were computed using an implementation (Huson and Wetzel 1994) of the Split Decomposition method of Bandelt and Dress (1992) which provides a visual representation of the support for contradictory patterns in the data which indicate "weakly compatible splits." For example, in Fig. 1 edge $e X$ and $e Z$ contradict each other but they are also held to be weakly compatible if $e X$ is greater in size than $e Y$. To represent contradictory support a SPLITSTREE graph allows a second dimension for internal edges instead of single line. Generally speaking, the more resolved (less contradicted) a signal appears in a data set the less box-like and more tree-like the graph becomes. Figure 3 shows that edge $e 2$ is very box-like in the cytochrome $b$ data, whereas it is a relatively strongly resolved edge in the D-loop data (see also Fig. 4 for comparison with bootstrap measures on neighbor joining trees)

for Jukes-Cantor and Kimura 2ST distance estimates/neighbor joining using MEGA 1.01 (Fig. 4C,D) (Kumar et al. 1993). Jackknifing has also been used to examine the effect of sample size on the LogDet spectra for eight taxa. That is, spectra were calculated for three randomly sampled D-loop subsets whose sequence length was equivalent to that of the cytochrome $b$ data set (120 sites). For this study with D-loop sequences, the base composition of the constant sites was retained and the varying sites were sampled without replacement in two separate experiments. In one, the final number of parsimony sites was maintained the same as in the cytochrome $b$ data set. In the second experiment the total number of varying sites was kept the same as in the cytochrome $b$ data set.

Maximum likelihood estimates of the phylogenetic relationships among the same set of eight taxa and the two outgroup sequences (Priapella olmecae and P. compressa) were made using DNAML3.5 (Felsenstein 1993). Cytochrome $b$ and D-loop data sets were again analyzed separately, and the support for Meyer et al.'s (1994) placement of the root on the edge $e 2$ was evaluated for the same four transformations and with bootstrapping. The Jukes-Cantor and Kimura 

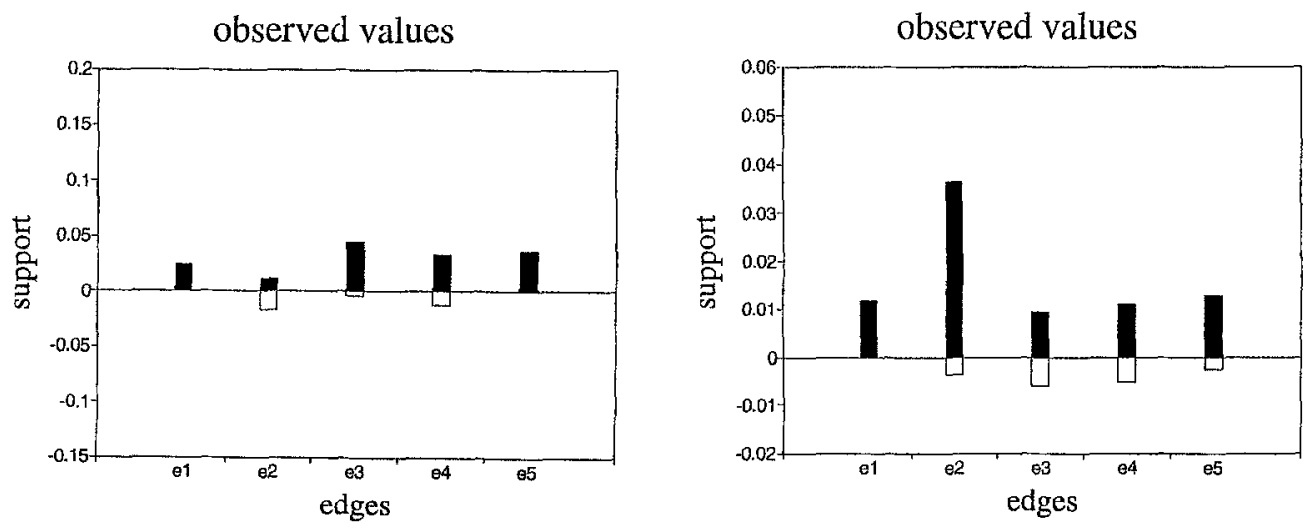

Jukes-Cantor values

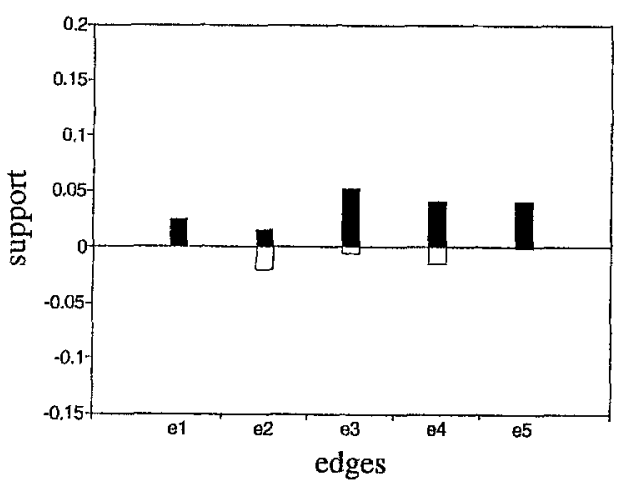

Jukes-Cantor values

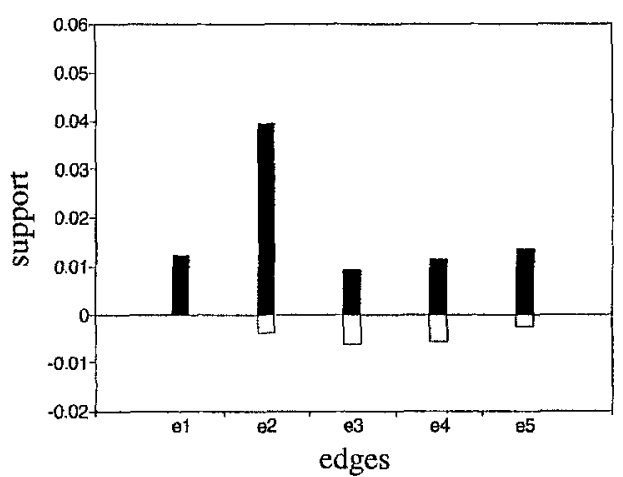

Kimura 2ST values

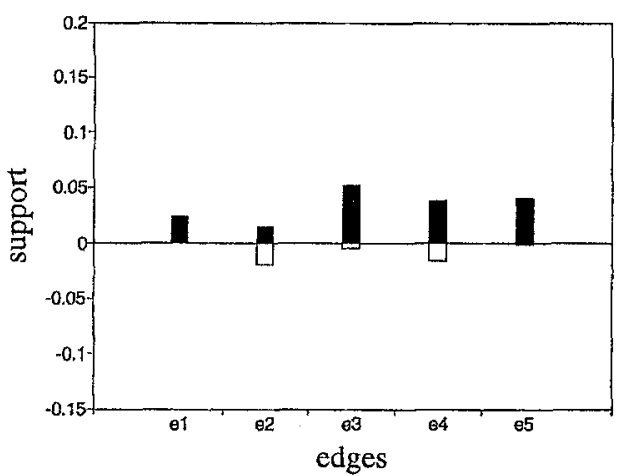

Kimura 2ST values

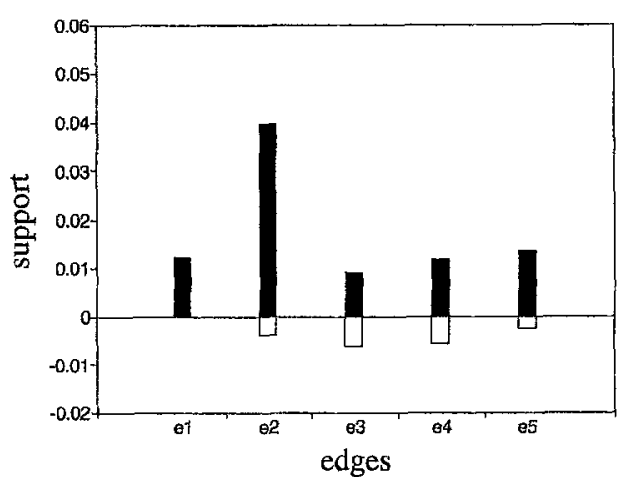

LogDet values

A
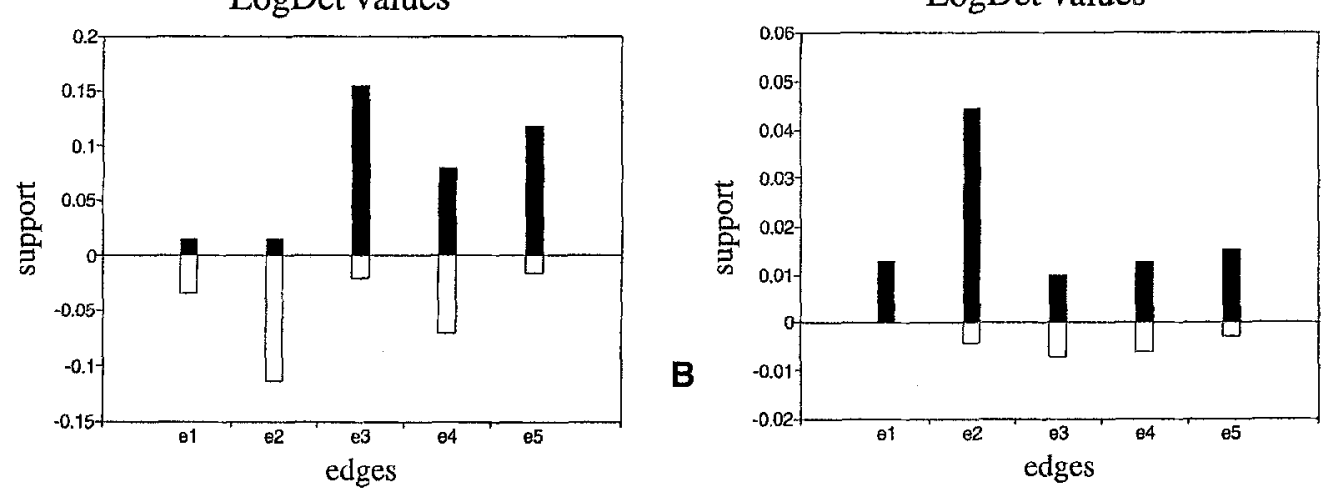

Fig. 4. These spectra indicate the support in eight cytochrome $b$ (A) and D-loop (B) sequences for the five internal edges of the Fig. 2 tree when the data are both untransformed (Hamming, observed) and transformed using simple correction formulae (i.e., Jukes-Cantor, Kimura 2ST, and LogDet). Above the zero point on the Y-axis is plotted the support for such edges. Below this point is plotted the sum of the conflicts in the data which would contradict each of these edges. The values for the partitions which identify these edges are calculated from the observed and transformed path measures between sequences using a Hadamard transformation (Hendy et al. 1994). For comparison,

bootstrap support for the edges el-e5 in cytochrome $b(\mathbf{C})$ and D-loop (D) data have also been calculated under neighbor joining when objective distances are estimated using Jukes-Cantor and Kimura 2ST for both ganma distributions (shape parameter $0.25,0.5,0.75,1,2$ ) and a constant rate of change across all sites. The respective \% bootstrap support for each edge is indicated in the figure. Very little difference in bootstrap values was found for both data sets when distances were estimated using a gamma distribution with shape parameters from 0.5 to 2.0 . 

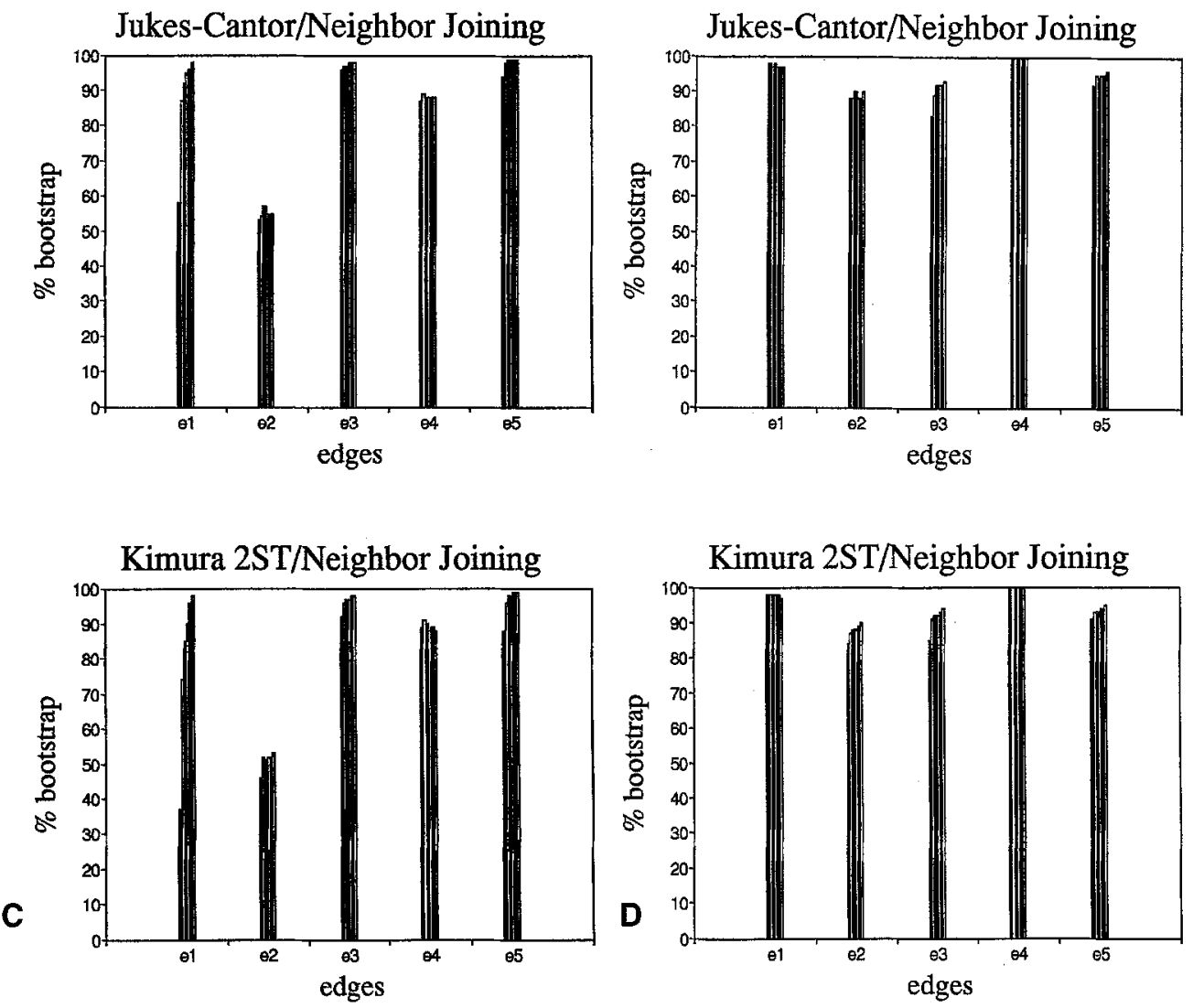

Fig. 4. Continued.

2ST transformations with neighbor joining have also been calculated using MEGA1.01 (Kumar et al. 1993). The spectral support calculated using HADTREE (Penny et al. 1993) for the internal edge partitions in the ten taxon spectra for Meyer et al.'s phylogeny is reported in Fig. 6.

\section{Results and Discussion}

\section{The Fit Between Data and Model}

If the relationships between taxa are well established then it is possible to examine how well sequence data determined from these species support the underlying phylogeny. For Xiphophorus fish such a phylogeny is shown in Fig. 2. The fit of the cytochrome $b$ and D-loop data to this phylogeny is examined in Figs. 3 and 4.

With the D-loop data, all internal edge partitions of the tree are well resolved (Fig. 3B and 4B) and transforming the data to correct for multiple changes has little effect on the spectral values (Fig. 4B). This presumably occurs because the data show little effect from multiple substitutions or base composition differences. Consequently, the observed patterns in the data (at least for this subset of taxa) are likely to be good indicators of phylogeny.

In contrast, the cytochrome $b$ data show unusual characteristics. An indication of this is given from the spectra, where transformation of the data using the LogDet algorithm dramatically changes the support for and against some edge partitions (Fig. 4A). This change occurs despite base compositions being similar in all compared cytochrome $b$ sequences. Hence, the different spectra do not result from the LogDet correcting for base composition differences as in Lockhart et al. (1994). Further, the LogDet transformation does not help resolve conflicts as would be expected if it better described the actual mechanism of sequence evolution for these data. The edge partition $\mathrm{e} 2$, which has high support in the D-loop data (Figs. 3B, 4B,D), is the smallest (and the edge with the most conflicts) in the cytochrome $b$ data (Figs. 3A, 4A,C). If the variances on the LogDet values were large as sample size became small then the different size of the cytochrome $b$ and D-loop data sets might explain the observed spectra. However, this was tested by randomly sampling D-loop positions to construct a data set the same length as that of the cytochrome $b$ data. Unlike the cytochrome $b$ data the subset D-Loop data still showed similar spectra for observed and LogDet values. This observation suggests that nucleotide substitution properties differ between the cytochrome $b$ and D-loop sequences.

\section{Rooting the Tree}

In a case where the patterns in sequence data do not strongly support the internal edges (i.e., cannot be used 


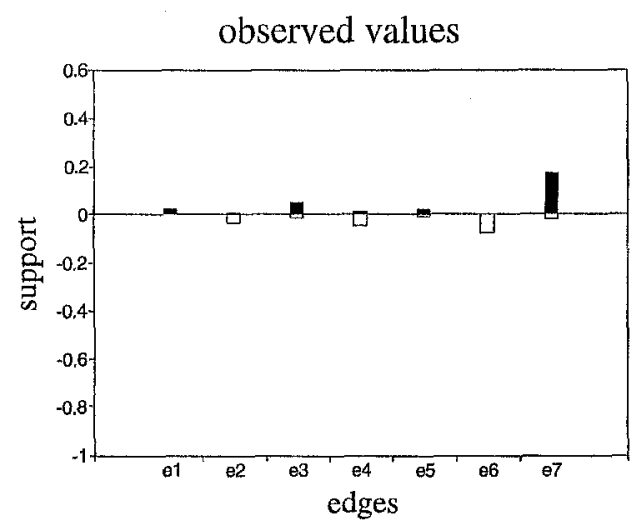

Jukes-Cantor values

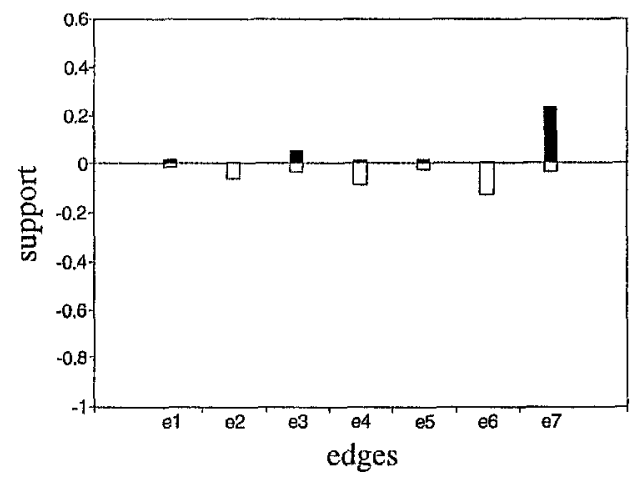

Kimura 2ST values

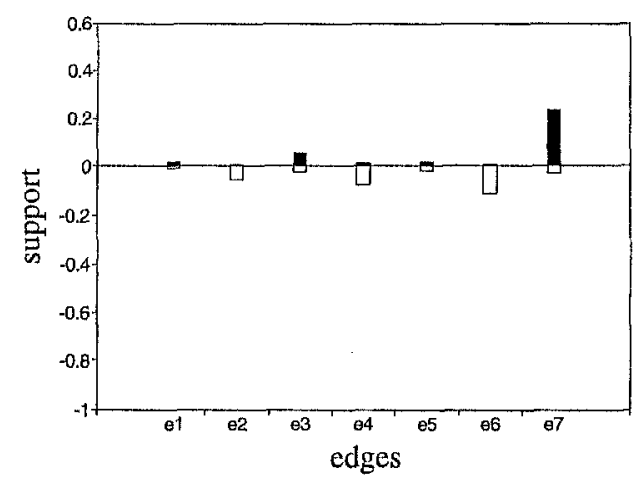

LogDet values

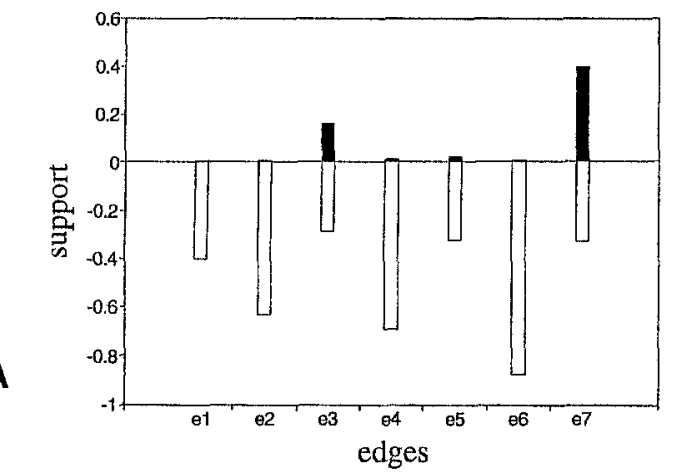

Fig. 5. Spectra for cytochrome $b$ (A) and D-loop (B) sequences for ten taxa which include the eight taxa shown in Fig. 2 plus two outgroup sequences. Above the zero point on the $Y$-axis is plotted the support for the internal edges that describe a hypothesis congruent with the D-loop tree. Below this point is plotted the sum of the patterns in the data which contradict each of these edges. For both cytochrome $b$ and D-loop sequences the spectra are presented in the same order as that

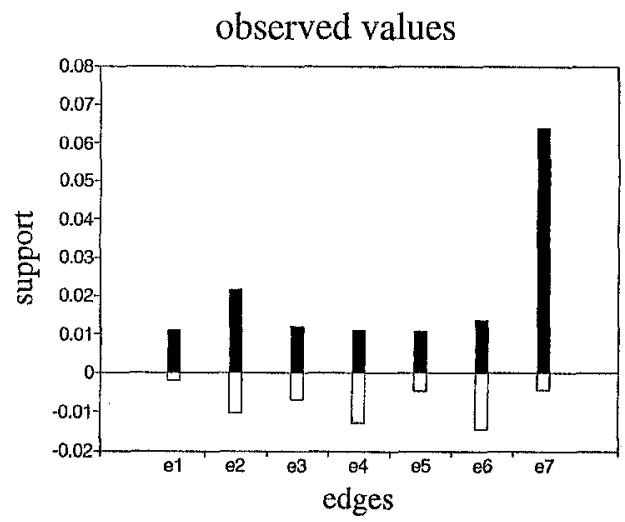

Jukes-Cantor values

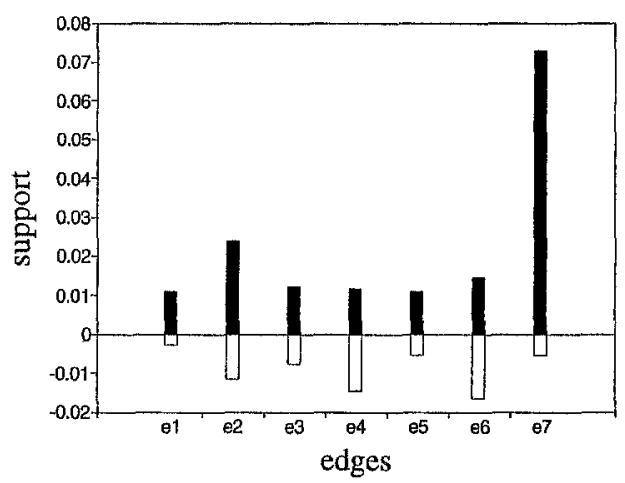

Kimura 2ST values

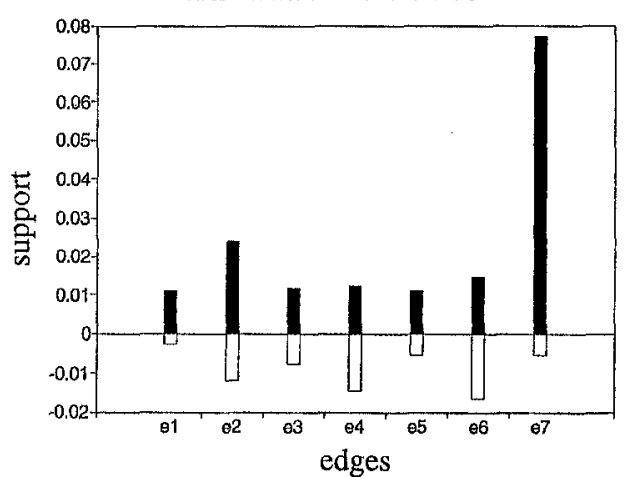

LogDet values

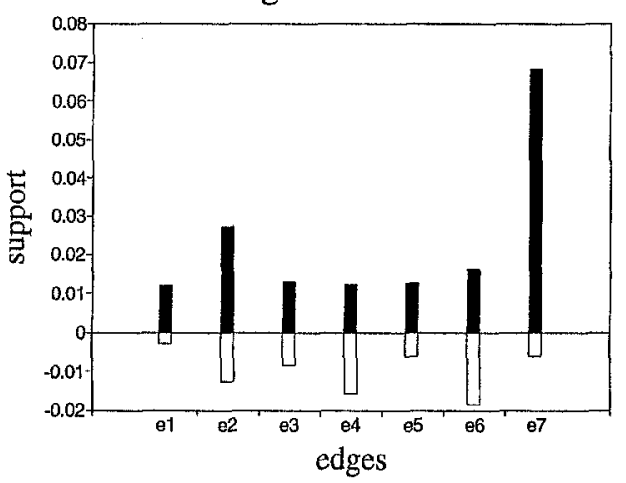

described in the legend to Fig. 4 -i.e., the spectra for Hamming distances, Jukes-Cantor distances, Kimura 2ST distances and LogDet values. The placement of the outgroups in the D-loop tree gives rise to two additional internal edges: $e 6$ and $e 7$ (Fig. 6B). In the observed cytochrome $b$ spectra $e 7$ is $-21 \times$ the size of $e 2$. In the D-Loop spectra it is $-3 \times$ the size of $e 2$. 
reliably to estimate the agreed-upon phylogeny) in an expected phylogeny it may be unrealistic to expect that addition of outgroup taxa (to help root that tree) could result in reliable inference. This follows from earlier observations on the problems of tree reconstruction when evolutionary trees are made up of long and short edges (Hendy and Penny 1989; Takezaki and Nei 1994).

Figure 2 shows the unrooted tree for a subset of the phylogeny recently proposed by Meyer et al. (1994). Fitting the observed data for both cytochrome $b$ and D-loop sequences to this tree requires that an outgroup edge-e7 joins edge $e 2$. With the D-loop data this is the optimal tree found using maximum likelihood (Fig. 6B), branch and bound "uncorrected"' parsimony (Steel et al. 1993b), and Neighbor Joining (on observed, JukesCantor, Kimura 2ST, LogDet values). In the case of neighbor joining with the Jukes Cantor or Kimura 2ST transformed data, bootstrap support for this outgroup placement was estimated to be between 89 and $94 \%$ (for gamma distribution shape parameters $0.5,0.75,1,2$, constant rate) (Fig. 4D). Confidence in this tree is also improved from the observation that the spectra for the D-loop observed or symmetrically corrected and LogDet transformed data is very similar (Figs. 4B, 5B). Since the LogDet is not yet implemented to accommodate different rates across sites and, further, since it has been suggested as being very susceptible to such effects (Lake, pers comm), this similarity of spectra together with the reported strong bootstrap results in Fig. 4C,D suggests that these D-loop data are sufficiently well described by the assumption that all sites in the sequences are changing independently, identically, and at the same rate. These assumptions are explicit in the implementation of the Jukes Cantor, Kimura 2ST, and LogDet transformations used in the spectra (Figs. 4, 5), SPLITSTREE graphs (Fig. 3), and also in the implementation of maximum likelihood used to reconstruct the tree in Fig. 6.

Given this apparent fit between these method assumptions and these D-loop data, the methods of tree reconstruction used here (all except uncorrected parsimony) should also correct for any additional long/short edge problem that might arise either because of inadequate sampling (Hendy and Penny 1989; Penny et al. 1994) or from rate differences between lineages (Felsentein 1978). Hence, with these D-Loop data and the methods of tree reconstruction used placement of the outgroup should be reliable.

However, with the cytochrome $b$ data, the spectra (Fig. 4) show that fitting the outgroup edge (e7) onto edge $e 2$ requires placing a very long edge onto a very short edge for which there is a large amount of internal conflict in the data (Fig. 4A,C; 5A and legend). In this case, because the pattern of sequence change in the eight sequences studied does not strongly support the expected unrooted phylogeny (possibly because the sequences are not changing independently or identically) the actual

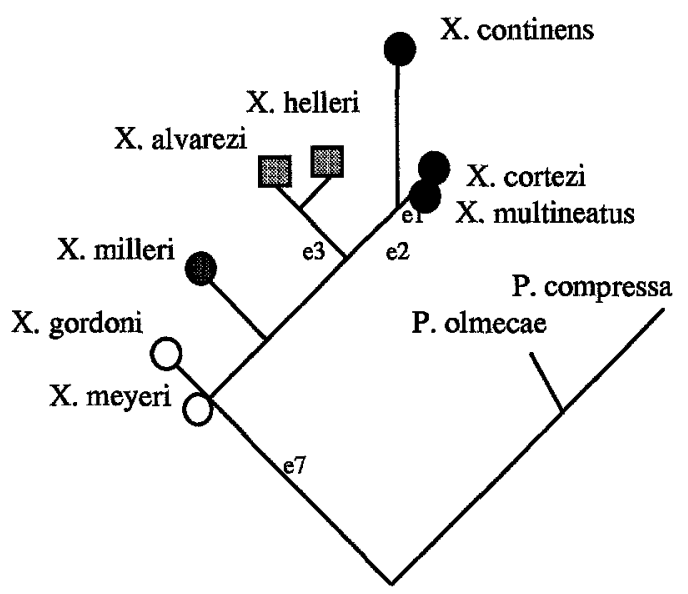

A

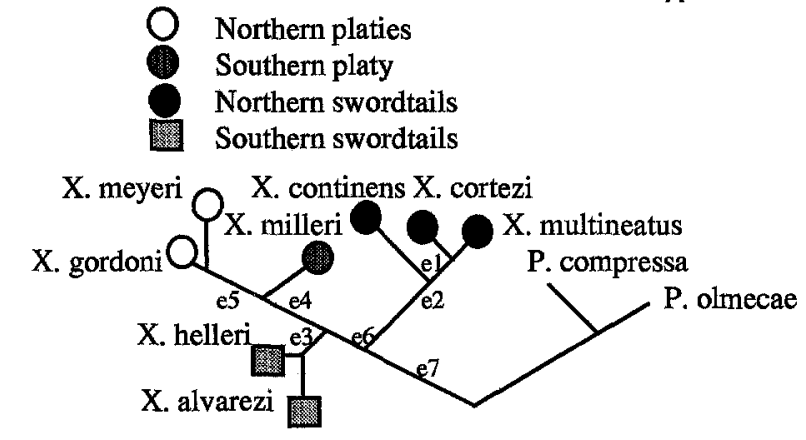

B

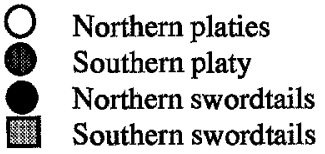

Fig. 6. Maximum likelihood estimate for the same eight species of Xiphophorus (as in Figs. 2,3) plus two outgroups made using DNAML $3.5(\mathrm{~T}=2$; Felsenstein 1993) for the evolutionary trees shown cytochrome $b$ (A) D-loop (B). The trees were reconstructed using two outgroup sequences and are drawn to suggest rooted phylogenies. The trees differ in the placement of the outgroups.

placement of the outgroup edge is likely to be unreliable. Consistent with this expectation, reconstructed trees from these cytochrome $b$ sequences using the same phylogenetic methods as here (which when applied to the D-loop data produced only one optimal unweighted tree) are also not congruent. The optimal tree reconstructed with cytochrome $b$ data using a maximum likelihood procedure is shown in Fig 6A. Bootstrap support measured for Jukes-Cantor and Kimura 2ST distances with neighbor joining also shows relatively low values supporting that root placement (Fig. 4C). Indeed as the shape parameter for gamma distributions is changed so does the edge onto which the outgroup joins. Such placements are either onto the $X$. meyeri edge (as in Fig. 6) or onto the edge separating $X$. meyeri and $X$. gordoni from the other taxa. For shape parameters between 0.5 and 2 bootstrap values range from 54 to $66 \%$ (Fig. 4C). 


\section{Cytochrome $b$ and D-loop Sequence Evolution}

We use the spectra for cytochrome $b$ and D-loop sequences to make inferences about the reliability of tree reconstruction from these two independent data sets. Since both the cytochrome $b$ and D-loop segments are widely used as phylogenetic markers (reviewed, e.g., in Meyer 1993, 1994a,b) in molecular systematics the results from the present study may also have general implications.

Our observations identify contradictory patterns in the cytochrome $b$ sequences which cannot be corrected for by using three simple mathematical transformations which correct for unobserved multiple substitutions (Figs. 4A, 5A). In contrast, the spectra, for the D-loop sequences from the same taxa, and using the same corrections as used in cytochrome $b$, show a much clearer resolution of compatible signals (Figs, 4B, 5B) that uniquely fit one evolutionary tree (Fig. 6B).

In their earlier study which included sequences from a much larger number of taxa (52 individuals sampled to give 36 representative sequences), the observations of Meyer et al. (1994) also support these findings. That is, using the same heuristic search options under uncorrected parsimony, these authors found that the D-loop sequence data gave far fewer optimal trees (six shortest trees all with the same root placement; length $335, \mathrm{Cl}=$ $0.633, \mathrm{RC}=0.482$ ) than did the cytochrome $b$ sequences (all codon positions considered; $100+$ shortest trees, almost half showed different root placements; length 285 steps, $\mathrm{Cl}=0.596, \mathrm{RC}=0.438$ ).

\section{Testing the Fit Between Model and Data}

Where possible, in other studies it may be advantageous to attempt to characterize the extent of the fit between model and data rather than to just place faith in the robustness of a given method. The approach adopted for examining fit in this communication has been to examine the net support (signal-contradiction; see Fig. 1) under different transformations for partitions that are known to occur in an undisputed tree. This approach may in some cases be preferable to one of predicting data (given a tree and an explicit mechanism of sequence evolution) and then comparing such simulated data to the experimentally determined biological sequence data. We have used this other approach elsewhere (e.g., Lockhart et al. 1992; Hendy et al. 1994) but find, as do others (GJ Olsen, pers comm; Goldman 1993), that such predicted data can often show a poor fit to the optimal tree without clades in the reconstructed tree being necessarily wrong.

In our present study we have used the LogDet transformation of Steel (1994) and examined the spectral patterns of an established phylogeny for the purpose of examining data-model fit. The LogDet's present implementation allows compared sequences to show irregular patterns of nucleotide frequency but it does require that all sites in these sequences change independently, identically, and with the same rate of change across sites. While these assumptions are also important for the implementation of other transformations used in our spectral study, the LogDet has been suggested to be particularly susceptible to deviations from these requirements. This subject still requires further detailed study.

In the context of this paper, the poor resolution of expected patterns in cytochrome $b$ data under all transformations tried suggests that the Xiphophorus cytochrome $b$ third codon positions may not be evolving such that all sites are changing independently and identically. This observation must bring into question the reliability of inference for these cytochrome $b$ sequences until it can be determined how such deviations will affect the different path-length estimates made using simple transformations. In contrast, given present understanding of tree-building methods and the apparent fit of data and model for the D-loop data, our findings support earlier conclusions by Meyer et al. for a revised phylogeny of Xiphophorus fishes.

Our results also support earlier concerns on phylogenetic inference from some other sequence data sets (Meyer 1993, 1994a,b; Avise 1994)-that because of different rates of change and processes of sequence evolution, some genes or portions of genes may be more useful and appropriate than others for testing particular phylogenetic hypotheses. In the present context this observation gives rise to an additional issue.

\section{Heterogeneity Between Data Sets and Joint Analyses}

The results from the present work highlight the question of whether different sequence data sets should be jointly analyzed or examined independently. In our study, the cytochrome $b$ and D-loop sequences produce very different spectra for the same taxa. Hence, it would seem inappropriate to jointly analyze these data. In fact, our observations suggest that inference from the Xiphophorus cytochrome $b$ data is misleading and hence for the moment should be discounted.

Since the reliability of methods of tree reconstruction of biological sequence data is still poorly understood we agree with the views expressed by Huelsenbeck et al. (1994). These authors point out that when data sets are combined jointly without addressing possible evolutionary heterogeneity there is a risk of phylogenetic estimates failing to accurately represent the history of the group in question.

In the present paper, we have used one study to demonstrate how recent methodology might be implemented to investigate the significance of observed heterogeneity. The approach should also be helpful in other studies where there is a need to evaluate both the substitution properties and potential of different genes for testing particular biological hypotheses. 
Acknowledgments. Special thanks to Mike Steel, Mike Hendy, and Peter Waddell for helpful comments on the manuscript and also to Daniel Huson and Rainer Wetzel for help with Split Decomposition analysis. Thanks to Daniel Huson, David Pollock, and Chris Simon for critical comments on the manuscript. This work was supported by NSF grants (BSR-9119867 and BSR-9107838) and a grant from the New Zealand Public Good Science Fund.

\section{References}

Avise JC (1994) Why one-kilobase sequences from mitochondrial DNA fail to solve the hoatzin phylogenetic enigma. Mol Phyl Evol 3:175-184

Bandelt H-J, Dress AWM (1992) A canonical decomposition theory for metrics on a finite set. Adv Math 92:47-105

Basolo AL (1990) Female preferences predates the evolution of swords in swordtail fish. Science 250:808-810

Felsenstein J (1978) Cases in which parsimony or compatibility methods will be positively misleading. Syst Zool 27:401-410

Felsenstein J (1993) PHYLIP3.5 (available from joe@genetics. washington.edu). University of Washington, Seattle, WA

Golding B (1993) Maximum-likelihood estimates of selection coefficients from DNA sequence data. Evolution 47:1420-1431

Goldman N (1993) Statistical tests of models of DNA substitution. J Mol Evol 36:182-198

Hendy MD, Penny D (1989) A framework for the quantitative study of evolutionary trees. Syst Zool 38:297--309

Hendy MD, Penny D (1993) Spectral analysis of phylogenetic data. J Classification 10:5-24

Hendy MD, Penny D, Steel MA (1994) A discrete Fourier analysis for evolutionary trees. Proc Natl Acad Sci USA 91:3339-3343

Hillis DM, Huelsenbeck JP, Cunningham CW (1994) Application and accuracy of molecular phylogenies. Science 264:671-677

Huelsenbeck JP, Swofford DL, Cunningham C, Bull JJ, Waddell PJ (1994) Is character weighting a panacea for the problem of data heterogeneity in phylogenetic analysis? Syst Biol 43:288-291

Huson D, Wetzel R. (1994) SPLITSTREE V1.0 (available from huson orwetzel@mathematik.uni-bielefeld.de) University of Bielefeld

Kumar S, Tamura K, Nei M (1993) MEGA: molecular evolutionary genetics analysis, version 1.01. The Pennsylvania State University, University Park, PA 16802

Lake $J$ (1994) Reconstructing evolutionary trees from DNA and protein sequences: paralinear distances. Proc Natl Acad Sci USA 91:14551459

Lento GM, Hickson RE, Chambers GK, Penny D (1995) Use of spectral analysis to test hypotheses on the origin of pinnipeds. Mol Biol Evol 12:2852

Lockhart PJ, Penny D, Hendy MD, Howe CJ, Beanland TJ, Larkum AWD (1992) Controversy on chloroplast origins. FEBS Lett 301: $127-131$

Lockhart PJ, Steel MA, Hendy MD, Penny D (1994) Recovering evolutionary trees under a more realistic model of sequence evolution. Mol Biol Evol 11:605-612
Meyer A, Morrissey JM, Schartl M (1994) Recurrent origin of a sexually selected trait in Xiphophorus fishes inferred from a molecular phylogeny. Nature 368:539-542

Meyer A (1993) Evolution of mitochondrial DNA in fishes. In: Hochachka $\mathrm{P}$, Mommsen $\mathrm{P}$ (eds) Biochemistry and molecular biology of fishes, vol 2. Elsevier, pp 1-38

Meyer A (1994a) Shortcomings of the cytochrome $b$ genes as a molecular marker. Trends Ecol Evol 9:278-280

Meyer A (1994b) DNA technology and phylogeny of fish: molecular phylogenetic studies of fish. In: Beaumont AR (ed) Genetics and evolution of aquatic organisms. Chapman and Hall, pp 219-249

Olsen GJ, Woese CR (1993) Ribosomal RNA: a key to phylogeny. FASEB J 7:113-123

Penny D, Watson EE, Hickson RE, Lockhart PJ (1993) Some recent progress with methods for evolutionary trees. $\mathrm{N} \mathrm{Z} \mathrm{J}$ Botany 31: 275-288 (PREPARE and HADTREE available from FARSIDE@ massey.ac.nz)

Penny D, Lockhart PJ, Steel MA, Hendy MD (1994) The role of models in reconstructing evolutionary trees. In: Scotland RW, Siebert DJ, Williams DM, (eds) Models in phylogeny reconstruction. Clarendon Press, Oxford, pp 52, 211-230

Pomianskowski A (1994) Swordplay and sensory bias. Nature 368: 494-495

Rauchenberger M, Kallman KD, Morizot DC (1990) Monophyly and geography of the Rio Panuco basin swordtails (genus Xiphophorus) with descriptions of four new species. Am Mus Nat Hist Novitater 2975:1-41

Rosen DE (1979) Fishes from the upland and intermontane basin of Guatemala: revisionary studies and comparative geography. Bull Am Mus Nat Hist 162:268-375

Saitou N, Nei M (1987) The neighbor joining method: a new method for reconstructing phylogenetic trees. Mol Biol Evol 4:406-425

Shaw K (1995) Phylogenetic tests of the sensory exploitation model of sexual selection. Trends Ecol Evol 10:117-120

Simon C, Frati F, Beckenbach A, Crespi B, Liu H, Flock P (1994) Evolution, weighting, and phylogenetic utility of mitochondrial gene sequences and a compilation of conserved polymerase reaction primers. Ann Entomol Soc Am 87:651-701

Steel MA, Lockhart PJ, Penny D (1993a) Confidence in evolutionary trees from biological sequence data. Nature 364:440-442

Steel MA, Hendy MD, Penny D (1993b) Parsimony can be consistent! Syst Biol 42:581-587

Steel MA (1994) Recovering a tree from the leaf colourations it generates under a Markov model. Appl Math Lett 7:19-24

Swofford DL (1993) Phylogenetic analysis using parsimony, version 3.1.1. Illinois Natural History Survey, Champaign, IL

Takezaki N, Nei M (1994) Inconsistency of the maximum parsimony method when the rate of nucleotide substitution is constant. J Mol Evol 39:210-218

Yang Z, Goldman N, Friday (1994) Comparison of models for nucleotide substitutions used in maximum-likelihood phylogenetic estimation. Mol Biol Evol 11:316-324 